\title{
Reiter's syndrome versus seronegative rheumatoid arthritis
}

\author{
A. T. MASI, AND S. L. FEIGENBAUM \\ From the Peoria School of Medicine, University of Illinois, College of Medicine, Peoria, Illinois, USA
}

Because of our longitudinal studies of early rheumatoid arthritis (RA) in young adults ${ }^{106,210,211}$ we have been asked to compare the findings in males with Reiter's syndrome (RS) with those in patients with seronegative RA or arthritis of unknown diagnosis.

\section{Patients and methods}

We present the results of a preliminary analysis of the cases of 38 younger males first diagnosed as having arthritis between the ages of 15 and 44 years, including 21 with RS and four with seronegative RA. At entry to the study and within six months of the first diagnosis 211 all the patients had negative tests for IgM rheumatoid factor by latex ${ }^{290}$ and sheep cell agglutination. Most were HLA-typed by the microlymphocytotoxicity method using 55 antisera with 25 specificities. ${ }^{234}$ The findings presented here

Table 1 Selected clinical features found at initial examination of 38 younger men with Reiter's syndrome $(R S)$, rheumatoid arthritis $(R A)$, or arthritis of unknown diagnosis (AUD), grouped by last diagnosis

\begin{tabular}{|c|c|c|c|c|c|}
\hline \multirow[t]{2}{*}{ Selected features } & \multicolumn{3}{|c|}{ Reiter's syndrome } & \multirow[b]{2}{*}{$\begin{array}{l}R A \\
(n=4)\end{array}$} & \multirow[b]{2}{*}{$\begin{array}{l}A U D \\
(n=13)\end{array}$} \\
\hline & $\begin{array}{l}\text { Com- } \\
\text { plete } \\
(n=6)\end{array}$ & $\begin{array}{l}\text { Incom- } \\
\text { plete } \\
(n=15)\end{array}$ & $\begin{array}{l}\text { Total } \\
(n=2 I)\end{array}$ & & \\
\hline $\begin{array}{l}\text { Mean age at onset } \\
\text { (years) } \\
<25 \text { years } \\
\text { Whites (versus blacks) } \\
\text { HLA B27-positive } \\
\text { Urethritis (history) } \\
\text { Conjunctivitis (history) } \\
\text { Conjunctivitis (exam) } \\
\text { Symmetry† } \\
\text { Radiographic } \\
\text { sacroiliitis } \$ \\
\text { Mean ESR (mm in } \\
1 \text { h) Westergren } \\
>60 \text { mm in } 1 \mathrm{~h}\end{array}$ & $\begin{array}{l}19 \\
6 \\
3 \\
3(5) \\
6 \\
6 \\
3 \\
4 \\
2(4)\end{array}$ & $\begin{array}{l}24 \\
10 \\
8 \\
8(12) \\
10 \\
1^{*} \\
0 \\
7 \\
5(6)\end{array}$ & $\begin{array}{l}23 \\
16 \\
11 \\
11(17) \\
16 \\
7 \\
3 \\
11 \\
7(10)\end{array}$ & $\begin{array}{r}28 \\
2 \\
2 \\
0 \\
1 \\
0 \\
0 \\
3\end{array}$ & $\begin{array}{l}30 \\
4 \\
6 \\
0(8) \\
0 \\
1^{*} \\
0 \\
5\end{array}$ \\
\hline
\end{tabular}

* Both patients either B7 or BW22 positive.

tOne pair within 4 joints and $2+$ pairs within $5+$ joints affected. $\ddagger$ Irregularity of joint margins and sclerosis (grade $2+$ ).

Numbers in parentheses show number of patients tested when this was less than the total.
(Table 1) are selective and are not entirely representative of the 500 or so variables recorded for the annual examination of each patient. 211

All diagnoses were made clinically by experienced rheumatologists without knowing the B27 type. RS ('complete') was diagnosed in patients presenting with arthritis, urethritis, and conjunctivitis-that is, the complete triad. Despite the absence of (early) erosions and of rheumatoid factor, RA was diagnosed in persistent, non-remitting symmetrical arthritis. Standardised criteria for RA were not used. However, four of the eventual 15 patients with 'incomplete' RS, all B27-positive, had initially beeno diagnosed as seronegative RA but presented with minor features of RS on follow-up and were subsequently included among 'incomplete' RS and referred to as 'transfer patients'.

The arthritis of unknown diagnosis (AUD) classification was usually applied to patients with less symmetric arthritis manifestations who had either a more acute onset or less joint swelling than in RA, often associated with precipitating factors, and a milder or remitted course, unlike the more progressive course of RA. Such patients are difficult to diagnose even though they may fulfill proposed criteria for $\mathrm{RA}^{259}$, and we classify them AUD. We recognised that patients diagnosed as RA and AUD may belong to the same disease spectrum.* Whites and blacks were about equal in each group, which reflects our patient population.

As might be expected, all the B27 carriers were clustered in the group of 'complete' and 'incomplete' RS. Nevertheless, it must be emphasised that patients are grouped in this analysis according to their last clinical diagnosis. Exclusion of 'transfer patients' would have yielded a B27 prevalence of only 7 $(53.8 \%)$ among the 13 patients originally diagnosed as RS and typed for this antigen. $\dagger$

*See paper by T. Bitter at page 84 .

†A lower degree of B27 association than usually reported, illustrating the difficulty of early clinical diagnosis among seronegative arthritides and the need for clinical classification criteria-Editor. 
Interestingly, all four of the retained RA patients, seronegative at entry, converted to seropositive on follow-up, whereas none of the RS or AUD patients converted. Thus the RA patients in this study were seropositive within a few years and the major comparison is between the RS and AUD groups.

\section{Results}

Table 1 shows selected clinical and laboratory findings among the patient groups. Objective arthritis was a criterion for patient entry into the study and is not listed. Urethritis by history was the next most common manifestation in the RS patients, occurring in $76 \%$ overall and in $10(67 \%)$ of the 15 incomplete RS subgroup. Urethritis was present in one patient with RA and in none with AUD. Conjunctivitis, either on examination or by history, was present in $14 \%$ and $33 \%$ of the total RS patients, respectively, and in one AUD patient.

We followed Professor T. Bitter's definitions when analysing the presence or absence of symmetry - that is, it was present when at least one pair of joints was affected in pauciarticular cases (up to four joints) and at least two pairs were involved in polyarticular cases (five or more joints affected). The difference between the groups in the proportions of those with symmetrical involvement is not significant.

Remission was defined for purposes of this study as complete absence of joint pain, tenderness, or swelling for at least one year before the last examination. Seven $(33 \%)$ of RS and five $(38 \%)$ of AUD patients satisfied these criteria over an average follow-up of five years, but none of the RA patients did so. None of these patients had peripheral joint erosions at entry to the study and none developed them on follow-up, which included annual posteroanterior radiographs of hands and wrists.

A single $30^{\circ}$ cephalad-inclined anteroposterior radiographic view of the sacroiliac joints was taken in B27-positive patients and their matched controls included in our previous study. ${ }^{234}$ The proportion of RS patients with definite radiographic sacroiliitis was somewhat higher among the B27-positives than among the negatives- $83 \%$ versus $50 \%$. The numbers of sacroiliac studies in the other groups were too few to draw conclusions. The Westergren ESR was highest at entry in the RS and lowest in the AUD patients. The difference was significant $(P \leqslant 0 \cdot 05)$.

Three of the patients had cardiac manifestations which may have been related to RS. One teenage black patient had a history of arrhythmia and died suddenly of pulmonary oedema while competing in sports. Aortic regurgitation was asymptomatic in one white patient in his 20 s but contributed to signs of cardiac failure in one black patient in his late 30s.

The degree of involvement of each affected joint was recorded at entry by either pain and tenderness or swelling on first examination, or from a clear-cut history. There was no significant difference between the mean number of joints affected at onset (by history) and those found at first examination. By history and at the initial examination-that is, within an average of less than six months from first diagnosis-the RS patients had a significant $(P \leqslant 0.05)$ predominance of lower joint swelling as compared with the AUD patients. The greater degree of joint swelling in the RS patients is consistent with their higher ESR. Fewer joints were affected in RS patients than seen usually in RA. RA, however, affects mainly women, and comparisons in these diseases should be made only among those in the sex group under study.

The results were also analysed in relation to HLA-B27. By history RS patients without B27 reported significantly more upper $(P \leqslant 0 \cdot 01)$, lower $(P \leqslant 0 \cdot 05)$, and total $(P \leqslant 0.001)$ affected joints than their B27-positive counterparts. These B27-negative RS patients also reported significantly more lower $(P \leqslant 0.05)$ and total $(P \leqslant 0.05)$ painful-tender joints than the AUD patients. On physical examination, however, these differences could not be confirmed. In particular we did not find any significant difference in the extent or the pattern of joint involvement between B27-positive and B27-negative RS patients. Definite radiographic sacroiliitis, however, tended to concentrate in the B27-positive RS patients.

At the last follow-up visit six (40\%) out of 15 patients with 'incomplete' RS were in complete spontaneous remission as opposed to only one $(16 \%)$ of six patients with 'complete' RS, but this difference is not significant

\section{Discussion}

This preliminary analysis of selected entry data on 21 male RS patients and 13 male seronegative AUD patients has yielded a few results which merit comment. The younger-adult onset of RS is emphasised, especially in contrast to the somewhat older age of patients with AUD. Whether any AUD patients will develop further manifestations of RS is not known. After an average of five years follow-up none has urethritis and $38 \%$ are in a complete articular remission. In the early stages of RS the articular pattern is difficult to differentiate from other seronegative arthritis syndromes.

Seronegative peripheral arthritis patients without 
B27, sacroiliac joint involvement, or other indicators of RS are difficult to classifiy, especially early in the disease. A fair proportion will probably become seropositive during the follow-up and develop typical RA. Others will remain seronegative with continuing joint activity and simulate RA to a greater or lesser extent. The prognosis in such cases tends to be better in men than in women. About $40 \%$ of the men with seronegative AUD entered a complete articular remission and their diagnosis is not likely be to clarified further.* It may be a forme fruste of RA, a variant of incomplete RS, or another host-conditioned reactive syndrome to an infectious agent or environmental stress factors.

One-third of the RS patients were in articular remission at the last evaluation, but no correlation was found between the presence or absence of B27 and remission of peripheral arthritis in this small sample. Five (46\%) out of 11 B27-positive and one $(16 \%)$ out of six B27-negative patients were in remission when last seen. Relative predominance of lower extremity involvement was found in RS by both history and examination at the first consultation.

\section{Analysis of selected features in females}

The preceding comparisons were restricted to males for reasons of more critical analysis, since RS occurs predominantly in males. ${ }^{11}$ Similar data on females, however, were available in our longitudinal studies. The criteria for entry to the study, diagnosis, and follow-up were the same for the females as for the males. Sixteen younger female seronegative arthritis patients were initially diagnosed as RA on clinical findings, but the diagnosis in four B27-positive cases was changed to spondyloarthropathy after HLA typing and other follow-up results. Their ages were $17,23,28$, and 32 years at onset. None had conjunctivitis but two had $3^{+}$and $4^{+}$asymptomatic radiographic sacroiliitis (RSI). The youngest patient was originally believed to have gonococcal arthritis in association with $\mathrm{RA}$, but the onset of RS may have been at that time. The two patients with RSI (ages 23 and 32 at onset) have had continuing symmetrical polyarthritis, whereas the other two had an asymmetric arthritis affecting mainly the lower extremities with remittances. These four patients with probable spondyloarthropathy were eliminated from the subsequent analyses of female RA and AUD patients.

Selected host and clinical features of 12 younger female seronegative RA patients and 41 with AUD

\footnotetext{
*Some of these cases might qualify for a diagnosis of 'persistent yet reversible asvmmetric oligoarthritis'-Editor.
}

are shown in Table 2. Their mean ages at onset are comparable to the male RA and AUD patients and significantly older than the RS cases. None of the eight remaining RA patients tested for B27 was positive but three $(10 \%)$ of the 30 AUD cases tested were B27 positive. All three B7 cases had either a history of conjunctivitis or evidence of urethritis or chronic cervicitis, compared with eight $(30 \%)$ of the remaining $27 \quad B 27$-negative patients $(P<0 \cdot 05)$. Another patient with HLA-B7 had a history of both conjunctivitis and urethritis. One or more of these four AUD patients with either B27 or B7 and a history of conjunctivitis or genitourinary tract inflammation may also have a spondyloarthropathy condition-for example, a forme fruste of RS rather than AUD, as clinically diagnosed. Their arthritis syndromes were characterised by asymmetry, predominance of lower extremity involvement, and intermittency of manifestations. Radiographs showed no sacroiliitis, and none had symptoms of spinal arthritis. Even if all eight spondyloarthropathy patients were considered to have RS they would still constitute a minority $(14 \%)$ of the 57 with seronegative arthritis, unlike the majority in younger males.

Female seronegative RA patients had a highere proportion of symmetrical arthritis $(75 \%)$ than the other groups, but the difference was not significant. The Westergren ESR in RA and AUD females was similar to that found in the males but, again, was lower than in the male RS patients and significantly lower in the AUD females $(\mathrm{P}<0 \cdot 01)$.

The mean numbers of joints affected in the RA and AUD females are shown in Table 3. The RA patients had significantly more involvement than the

Table 2 Selected host and clinical features found at initial examination in 53 younger females with seronegative rheumatoid arthritis $(R A)$ or arthritis of unknown diagnosis $(A U D)$

\begin{tabular}{lll}
\hline Selected features & $\begin{array}{l}R A \\
(n=12)\end{array}$ & $\begin{array}{l}A U D \\
(n=41)\end{array}$ \\
\hline Mean age at onset (years) & 31 & 32 \\
$\quad$ 25 years & 3 & 10 \\
Whites (versus blacks) & 8 & 17 \\
HLA-B27 positive & $0(8)$ & $3(30)$ \\
HLA-B7 or BW22 positive* & $3(8)$ & $6(27)$ \\
Urethritis cervicitis (history) & 0 & 3 \\
Conjunctivitis (history) & 1 & 9 \\
Conjunctivitis (exam) & 0 & 1 \\
Symmetry $\dagger$ & 9 & 19 \\
Mean ESR (mm in 1 h) & & 21 \\
$\quad$ Westergren & 21 & 21 \\
$\geqslant 60$ min in 1 h & 1 & 3 \\
\hline
\end{tabular}

*Applies only to HLA-B27-negative patients.

tOne pair within 4 joints and $2+$ pairs within $5+$ joints affected.

Numbers in parentheses show number of patients tested when this was less than the total. 
Table 3 Mean numbers of joints affected as reported in the patient's history and as found on examination in 53 females with seronegative rheumatoid arthritis $(R A)$ and arthritis of unknown diagnosis $(A U D)$

\begin{tabular}{|c|c|c|c|c|c|c|}
\hline \multirow{2}{*}{$\begin{array}{l}\text { Type of joint } \\
\text { involvement }\end{array}$} & \multicolumn{3}{|l|}{ History } & \multicolumn{3}{|c|}{ Examination } \\
\hline & $\begin{array}{l}R A \\
(n=12)\end{array}$ & $\begin{array}{l}A U D \\
(n=4 I)\end{array}$ & $\begin{array}{l}P \\
\text { value }\end{array}$ & $\begin{array}{l}R A \\
(n=12)\end{array}$ & $\begin{array}{l}A U D \\
(n=4 I)\end{array}$ & $\begin{array}{l}P \\
\text { value }\end{array}$ \\
\hline $\begin{array}{l}\text { Pain/tender } \\
\text { Upper } \\
\text { Lower } \\
\text { Swelling } \\
\text { Upper } \\
\text { Lower }\end{array}$ & $\begin{array}{r}12 \cdot 3 \\
9 \cdot 2 \\
3 \cdot 1 \\
6 \cdot 4 \\
4 \cdot 1 \\
2 \cdot 3\end{array}$ & $\begin{array}{l}7 \cdot 3 \\
4 \cdot 3 \\
3 \cdot 0 \\
3 \cdot 7 \\
1 \cdot 8 \\
1 \cdot 9\end{array}$ & $\begin{array}{l}\text { NS } \\
<0 \cdot 05 \\
\text { NS } \\
\text { NS } \\
\text { NS } \\
\text { NS }\end{array}$ & $\begin{array}{l}13 \cdot 5 \\
8 \cdot 5 \\
5 \cdot 0 \\
3 \cdot 1 \\
2 \cdot 8 \\
0 \cdot 3\end{array}$ & $\begin{array}{l}5 \cdot 4 \\
3 \cdot 8 \\
1 \cdot 6 \\
0 \cdot 8 \\
0 \cdot 5 \\
0 \cdot 3\end{array}$ & $\begin{array}{l}<0.01 \\
<0.05 \\
<0.001 \\
<0.01 \\
<0.001 \\
\text { NS }\end{array}$ \\
\hline
\end{tabular}

AUD cases, particularly of the upper extremity joints. The involvement in the AUD females was similar to that in the AUD males, The proportion of upper to lower joints swollen was greater than 1.0 in both groups of females, unlike the findings in male RS cases.

We may assume that RS is more common in females than is generally suspected, but it seems to be less acute than in males-perhaps analogous to ankylosing spondylitis. ${ }^{11}$ Among younger female seronegative arthritis patients with B27 we may suspect an even higher relative incidence of atypical arthritis syndromes which exhibit clinical mani- festations of RS-for example, asymmetric or intermittent involvement affecting lower extremity joints predominantly and associated with manifestations of conjunctivitis or urinary tract inflammation.

\section{General discussion}

DR. G. R. V. HUGHES: Have you, or has anyone else you know of, specifically looked at IgG rheumatoid factors in both RS and seronegative RA?

PROF. MASI: We have not done so and I don't know of anybody who has. Perhaps Morris Ziff might comment on that?

PROF. M. ZIFF: The IgG rheumatoid factors have been looked for in seronegative diseases by measurements that nobody trusts. They have been found to be increased in psoriatic arthritis, in RS, in juvenile chronic arthritis. The technique is that of immunodiffusion against aggregated IgG, which is notoriously unreliable. Better data might be forthcoming now that there is a radioimmunoassay available.

PROF. R. F. WILLKENS: Using the new radioimmunoassay techniques, Dr. Stage is actually engaged in such research. He feels that the methodologies in the past have been inaccurate. He has found no populations of seronegative arthritis patients that clearly have IgG rheumatoid factors. 